\title{
Diagnostic value of fine needle aspiration biopsy in lymphadenopathy of the head and neck
}

\author{
Karen Dzhabalyan', Tsvetan Tonchev', Nedyalka Zgurova², Ivan Krasnaliev² \\ 'Department of Oral and Maxillofacial Surgery and Special Imaging Diagnostics, Medical University - Varna \\ ${ }^{2}$ Department of General and Clinical Pathology, Forensic Medicine and Deontology, Medical University - Varna
}

\begin{abstract}
Lymph nodes in the head and neck region are involved in different pathological processes - metastatic, lypmhoproliferative and inflammatory. The objective of the present study was to evaluate the diagnostic accuracy of fine needle aspiration biopsy (FNAB) and define its place in the diagnostic algorithm. The results of the cytological evaluation of FNAB, confirmed with histological evaluation show that $72.8 \%$ of space-occupying lesions of the lymph nodes were malignant, with $48.2 \%$ being metastatic. Non-Hodgkin
\end{abstract}

lymphoma represented $69.1 \%$ of all lymphomas, whereas $28.3 \%$ were diagnosed as Hodgkin. Benign processes were represented almost exclusively by lymphadenitis $-93.9 \%$. Sensitivity of malignancy was high $(92.6 \%)$. FNAB had an accuracy of $79 \%$. Specificity was low at $45.5 \%$, mostly on account of lymphadenitis.

Key words: Lymph nodes, head and neck, fine needle aspiration biopsy

\section{Introduction}

Lymph nodes (LN) in the head and neck region are involved in different pathological processes - inflammatory, neoplastic and metastatic. The most commonly found malignant cell material from lymph nodes in the head and neck region represents regional metastasis from spinocellular carcinoma (SCC). ${ }^{1}$ Distant metastases in the head and neck region are relatively rare, keeping in mind that most authors agree that only $1 \%$ of carcinomas in the head and neck region can be classified as non-regional metastasis. ${ }^{2}$ Lymphomas account for $12 \%$ to $15 \%$ of the malignant tumors in the head and neck region. ${ }^{3,4}$ Cervical lymphadenopathy is the first clinical sign in $70 \%$ of cases, while the frequency of extranodal lymphoma is second in the head and neck region. ${ }^{5}$ Non-Hodgkin lymphomas comprise $75 \%$ of the head and neck lymphomas, ${ }^{6}$ with extranodal localization in $23 \%-30 \%$ of cases. ${ }^{7}$ According to Howlett et al. ${ }^{8}$ and Gonzales et al. ${ }^{9}$ around $30 \%$ of head and neck masses are a manifestation of reactive nodular hyperplasia and/or lymphadenitis. Granulomatous disease represents a sporadic event.

Use of imaging techniques as a single method of examination is insufficient in determining the character of disease. ${ }^{8}$ Fine needle aspiration biopsy is an easy to perform and minimally invasive method to collect cell material for cytological examination. ${ }^{9}$ The lymph nodes in the head and neck area are relatively easy accessible for FNAB. In cases of deep localization or small size, it is appropriate to use ultrasound navigation while performing FNAB. According to Rodjan et al. ${ }^{10}$ ultrasound-guided FNAB has great advantages in the detection of early stage SCC and clinically nonpalpable lymph nodes (4-5 mm diameter). The diagnostic workup of other malignant neoplasms, e.g. affecting mammary and thyroid glands, includes ultrasound and FNAB. ${ }^{11}$ Current research suggests FNAB as a specific diagnostic tool on suspicion of a cervical metastasis 
from a cancer unknown primary (CUP). ${ }^{12,13}$ Moreover, according to Balm et al. ${ }^{13}$ only subsequent negative or nondiagnostic FNABs are an indication for incisional, excisional or tru-cut biopsy.

The aim of our research is to evaluate the diagnostic value of FNAB and to define its place in the diagnostic treatment algorithm.

\section{Materials and Methods}

\subsection{Examined contingent}

The present research was approved by the ethics commission of Medical University of Varna. For the period between 2008 and 2014 in the division of Maxillofacial surgery (University Hospital "Sv. Marina") were tracked 146 patients with lymph node pathology of the head and neck region, which underwent FNAB. In 13 cases the results were uninformative, which necessitated a second biopsy. The present research included 114 patients with diagnostic cytology, which were treated surgically. The cohort included 65 men (57\%) and 49 women $(43 \%)$ aged between 4 and 95 , with the highest rate of morbidity being in the sixth and seventh decade.

\subsection{FNAB execution}

FNAB was performed using disposable needles size $25(0.5 \mathrm{~mm}$ Ч $38 \mathrm{~mm} / 25$ Gauge Ч $11 / 2)$ and disposable syringes with a volume of 2 to $5 \mathrm{ml}$ and freehand aspiration technique. Cell material was extracted from the center and periphery of the mass.

\subsection{Cytopreparatory technique}

The extracted cell material was applied to a slide, which was pushed into another with a sliding motion. This way the suspension was distributed evenly and four smears were obtained ( 2 center and 2 periphery smears). The smears were left to air dry for an hour, fixed in methanol for five minutes and subsequently dyed with Giemsa (methylene blue, eosin, and Azure B).

\subsection{Diagnostic categories of FNAB}

Diagnostic categories of FNAB stratified the risk of malignancy. The diagnostic classification concerning the assessment of FNAB was conducted in six categories: 0 - non-diagnostic; I - definitely benign; II - probably benign; III - possibly malignant; IV - probably malignant; V - definitely malignant.

The applied diagnostic categorization used two negative for malignant process categories, I and II, respectively definitely benign and probably benign. Three positive categories stratified the risk of a malignant process - III, IV, V, respectively possibly malignant, probably malignant and definitely malignant.

\subsection{Statistical Analysis}

Data were analyzed by the means of descriptive, non-parametric tests - chi-square test, cross-tabulation (IBM SPSS Statistics for Windows, v. 21.0, Armonk, NY, USA). The significance level was adjusted at $P<0.05$.

Sensitivity, specificity, diagnostic accuracy, the positive predictive value (PPV) and the negative predictive value (NPV) of FNAB were calculated. Sensitivity was defined as the percentage of patients who were correctly diagnosed to have malignancy on FNAB. Specificity was defined as the percentage of patients who were correctly diagnosed to have benign disease on FNAB.

\section{Results}

FNAB was non-diagnostic in $8.9 \%$ of cases (13 out of 146). Histologic verification of the results of FNAB showed that $72.8 \%$ of space-occupying lesions are malignant. Lymph node metastasis represented about half (48.2\%) of all malignant neoplastic processes (Table 1). Among these, SCC was most common $-48.7 \%(n=19)$ of all metastatic tumors, followed by poorly differentiated carcinoma $-7.7 \%(\mathrm{n}=3)$, undifferentiated carcinoma $-7.7 \%(\mathrm{n}=3)$ and malignant melanoma $-7.7 \%(\mathrm{n}$ $=3)$, metastasis from adenocarcinoma $(\mathrm{n}=2)$ and mucoepidermoid carcinoma $(\mathrm{n}=2)$. Non-Hodgkin/Hodgkin lymphoma represented, respectively, $69.1 \%$ and $28.6 \%$ of the malignant lymphoproliferative disorders. Lymphadenitis accounted for $94.9 \%$ of all space-occupying benign lesions, while only two cases of lymphangioma were noted. 


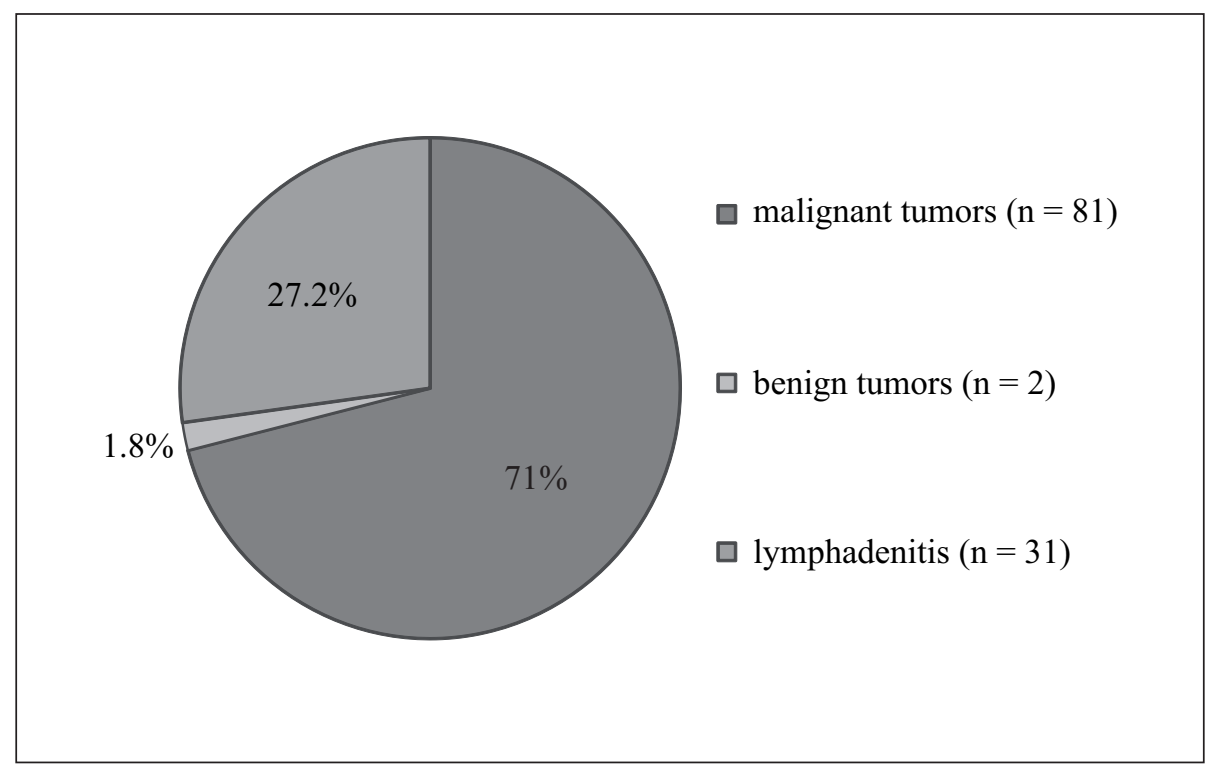

Figure 1. Lymph node pathology as confirmed by histological examination

Table 1. Histopathology of the lymph nodes

\begin{tabular}{|l|l|c|}
\hline & \multicolumn{1}{|c|}{ Histological classification } & Number \\
\hline Metastatic tumours & 1. Squamous-cell carcinoma & 19 \\
\hline & 2. Poorly differentiated carcinoma & 3 \\
\hline & 3. Undifferentiated carcinoma & 3 \\
\hline & 4. Malignant melanoma & 3 \\
\hline & 5. Poorly differentiated adenocarcinoma & 2 \\
\hline & 6. Mucoepidermoid carcinoma & 2 \\
\hline & 7. Papillary carcinoma & 2 \\
\hline & 7. Basal cell adenocarcinoma & 1 \\
\hline & 8. Small cell carcinoma & 1 \\
\hline & 9. Mesothelioma & 1 \\
\hline & 10. Medullary carcinoma & 1 \\
\hline & 13. Neuroendocrine carcinoma & 1 \\
\hline Lymphoma & & \\
\hline & & 29 \\
\hline & 1. Non-Hodgkin & 12 \\
\hline Benign neoplasms & 2. Hodgkin & 1 \\
\hline Inflammatory disorders & 3. Other malignant lymphoproliferative processes & \\
\hline & Lymphangioma & 31 \\
\hline
\end{tabular}

The statistical analysis showed that the extracted data was statistically significant $(P<0.05)$. Sensitivity and diagnostic accuracy are, respectively, $92.6 \%$ and $79 \%$. The most common false negative results (5) were found with lymphoma. Interestingly the specificity is only $45.5 \%$. This was mainly due to the fact that 17 out of 18 false positive results are from FNAB of lymphadenitis.
Table 2. Diagnostic value of FNAB of lymph node lesions

\begin{tabular}{|l|r|}
\hline TP $(n)$ & 75 \\
\hline FP $(n)$ & 18 \\
\hline TN $(n)$ & 15 \\
\hline FN $(n)$ & 6 \\
\hline PPV (\%) & 80.7 \\
\hline NPV (\%) & 71.4 \\
\hline Sensitivity (\%) & 92.6 \\
\hline Specificity (\%) & 45.5 \\
\hline Diagnostic accuracy (\%) & 79 \\
\hline
\end{tabular}




\section{Discussion}

In the present study $8.9 \%$ of FNABs were nondiagnostic. Other autors came to similar results. ${ }^{14}$ The most common reason for low diagnostic yield are metastases (from SCC, thyroid cancer, malignant melanoma) ${ }^{13}$ with cystic degeneration, the cyst wall being lined by relatively mature, benignappearing squamous cells. ${ }^{14}$ FNAB in these cases can turn out non-diagnostic, due to aspiration of centrally located cystic fluid, which is evacuated mostly because of its lower resistance. ${ }^{14}$ Another reason for a non-diagnostic FNAB are fibrous lesions, which frequently do not provide enough cell material for interpretation, due to difficulties with the aspiration. ${ }^{15}$ Lesions with high of blood volume, presence of necrosis and fibers with scattered atypical cells are also difficult for cytological analysis. ${ }^{15}$

Publications about sensitivity for malignancies in the head and neck region are few as a whole and/or do not provide separate data about the diagnostic accuracy of FNAB of lymph nodes. The majority of autors discuss staging of oral and oropharyngeal SCC. ${ }^{12,14,16,17}$ A study of Souren et al., ${ }^{14}$ comparing FNAB, ultrasound and magnet resonance imaging for staging of oral and oropharyngeal SCC, reported a high sensitivity (81\%) and specificity (100\%) of ultrasound guided FNAB of lymph nodes. In a similar study Geetha et al. ${ }^{16}$ report $67 \%$ sensitivity and $100 \%$ specificity. These results are not entirely comparable with the present outcome, because our cohort had unspecified lymphadenopathy at initial presentation. Nonetheless, in the present investigation all metastases were recognized correctly by FNAB as malignant (sensitivity 100\%) and the overall sensitivity for malignant process was high $-92.6 \%$. Despite the presence of five false negative results (predominantly FNAB of lymphoma), the sensitivity for lymphoma was high $-88 \%$. Karimi-Yazidi et al. ${ }^{18}$ confirm these observations. They reported a sensitivity of $88 \%$ and a specificity of $82.1 \%$. A possible reason for a false negative result can be the presence of ReedSternberg cells, which on rare occasions can be found not only in Hodgkin, but also in Non-Hodgkin lymphoma (after initial treatment), viral infections, such as infectious mononucleosis as well. ${ }^{7}$ Moreover, FNAB is problematic in the differentiation of a low-grade Non-Hodgkin lymphoma from reactive nodal hyperplasia. ${ }^{7}$ Flow cytometry in combination with FNAB can increase the diagnostic accuracy in these cases. ${ }^{7}$ Nonetheless, the application of FNAB in lymphoma remains controversial and many pathologists use it only for the diagnosis of relapse or in the most obvious cases of lymphoma. ${ }^{19}$

In the present study more than half of the benign processes (lymphadenitis and lymphangioma) were recognized as malignant -17 out of all 18 false positive results were from FNAB of inflammatory disorders of the lymph nodes, resulting in a low specificity of $45.5 \%$. Koo et al. ${ }^{20}$ reported that metastatic lymph nodes (e. g. nasopharyngeal carcinoma, pulmonary small cell carcinoma, papillary thyroid carcinoma, malignant melanoma and rhabdomyosarcoma) have the characteristics of a granulomatous inflammation. This can be attributed to necrotic tissue response or T-cell mediated hypersensitivity to antigens. ${ }^{20}$

Contingent selection undergoing FNAB could lead to a substantial increase of specificity. Inclusion of patients only suspicious for malignancy or for restaging would exclude the majority of inflammatory lymphadenopathies. On the other hand, the application of FNAB in all patients with lymphadenitis can be advantageous. A recent study ${ }^{21}$ showed that $45 \%$ of the cases of FNAB of lymph nodes represent a non-specific inflammatory lymphadenopathy, not necessitating inpatient treatment. The same investigation showed that in children this percentage is $85 \%$. Other authors report that the application of FNAB can reduce excisional/incisional biopsy by $40 \%-75 \%$ in pediatric head and neck masses. ${ }^{22}$

Fine needle aspiration cytology is associated with less complications than excisional lymph node biopsy, the latter having a complication rate of $5 \%$ to $10 \% .{ }^{11}$ Moreover, FNAB can be conducted without anesthesia and reduce treatment costs for staging of malignant disease ${ }^{20}$ with up to $55 \%$ in comparison to excisional lymph node biopsy. ${ }^{11}$ It can be concluded that FNAB is highly recommended in the diagnostic work-up of head and neck lymphadenopathy, avoiding unnecessary hospitalization in many cases.

\section{References:}

1. Pusztaszeri MP, Faquin WC. Cytologic evaluation of cervical lymph node metastases from cancers of unknown primary origin. Seminars in Diagnostic Pathology 2015; 32(1): 32-41.

2. McClure SA, Movahed R, Salama A, Ord RA. Maxillofacial metastases: a retrospective review of one institution's 15-year experience. J Oral Maxillofac Surg 2013; 71(1): 178-88. 
3. Cooper JS, Porter K, Mallin K, et al. National Cancer Database report on cancer of the head and neck: 10-year update. Head \& neck 2009; 31(6): 748-58.

4. Iguchi H, Wada T, Matsushita N, Oishi M, Yamane H. Anatomic distribution of hematolymphoid malignancies in the head and neck: 7 years of experience with 122 patients in a single institution. Acta oto-laryngologica 2012; 132(11): 1224-31.

5. Behrbohm H, Kaschke O. Ear, nose, and throat diseases: with head and neck surgery: Thieme; 2009.

6. Flint PW. Cummings otolaryngology--head \& neck surgery. Sixth edition. ed. Philadelphia, PA: Elsevier/Saunders; 2015.

7. Herd MK, Woods M, Anand R, Habib A, Brennan PA. Lymphoma presenting in the neck: current concepts in diagnosis. $\mathrm{Br}$ J Oral Maxillofac Surg 2012; 50(4): 309-13.

8. Saha S, Woodhouse NR, Gok G, Ramesar K, Moody A, Howlett DC. Ultrasound guided Core Biopsy, Fine Needle Aspiration Cytology and Surgical Excision Biopsy in the diagnosis of metastatic squamous cell carcinoma in the head and neck: an eleven year experience. Eur J Radiol 2011; 80(3): 792-5.

9. Mehanna H, McQueen A, Robinson M, Paleri V. Salivary gland swellings. BMJ 2012; 345: e6794.

10. Rodjan F, de Bree R, Weijs J, Knol DL, Leemans CR, Castelijns JA. Refinement of selection criteria to perform ultrasound guided aspiration cytology during follow-up in patients with early staged oral cavity carcinoma and initially cN0 necks. Oral Oncol 2011; 47(5): 391-4.

11. Voit CA, Gooskens SL, Siegel P, et al. Ultrasound-guided fine needle aspiration cytology as an addendum to sentinel lymph node biopsy can perfect the staging strategy in melanoma patients. Eur J Cancer 2014; 50(13): 2280-8.

12. Strojan P, Ferlito A, Medina JE, et al. Contemporary management of lymph node metastases from an unknown primary to the neck: I. A review of diagnostic approaches. Head \& neck 2013; 35(1): 123-32.

13. Balm AJ, van Velthuysen ML, Hoebers FJ, Vogel WV, van den Brekel MW. Diagnosis and treatment of a neck node swelling suspicious for a malignancy: an algorithmic approach. International journal of surgical oncology 2010; 2010: 581540.
14. Souren C, Kloss-Brandstatter A, Stadler A, et al. Ultrasoundguided fine-needle aspiration cytology as a diagnostic tool in comparison to ultrasound and MRI for staging in oral- and oropharyngeal squamous cell tumors. J Craniomaxillofac Surg 2015.

15. Rathod GB, Parmar P. Fine needle aspiration cytology of swellings of head and neck region. Indian journal of medical sciences 2012; 66(3-4): 49-54

16. Geetha NT, Hallur N, Goudar G, Sikkerimath BC, Gudi SS. Cervical lymph node metastasis in oral squamous carcinoma preoperative assessment and histopathology after neck dissection. Journal of maxillofacial and oral surgery 2010; 9(1): 42-7.

17. Stoeckli SJ, Haerle SK, Strobel K, Haile SR, Hany TF, Schuknecht $B$. Initial staging of the neck in head and neck squamous cell carcinoma: A comparison of CT, PET/CT, and ultrasound-guided fine-needle aspiration cytology. Head \& neck 2012; 34(4): 46976.

18. Karimi-Yazdi A, Motiee-Langroudi M, Saedi B, et al. Diagnostic value of fine-needle aspiration in head and neck lymphoma: a cross-sectional study. Indian journal of otolaryngology and head and neck surgery : official publication of the Association of Otolaryngologists of India 2014; 66(Suppl 1): 277-80.

19. Roh JL, Huh J, Moon HN. Lymphomas of the head and neck in the pediatric population. Int J Pediatr Otorhinolaryngol 2007; 71(9): 1471-7.

20. Koo V, Lioe TF, Spence RA. Fine needle aspiration cytology (FNAC) in the diagnosis of granulomatous lymphadenitis. The Ulster medical journal 2006; 75(1): 59-64.

21. Moor JW, Murray P, Inwood J, Gouldesbrough D, Bem C. Diagnostic biopsy of lymph nodes of the neck, axilla and groin: rhyme, reason or chance? Annals of the Royal College of Surgeons of England 2008; 90(3): 221-5.

22. Anne S, Teot LA, Mandell DL. Fine needle aspiration biopsy: role in diagnosis of pediatric head and neck masses. Int J Pediatr Otorhinolaryngol 2008; 72(10): 1547-53.

\section{Corresponding author:}

Karen Dzhabalyan

Department of Oral and Maxillofacial Surgery and Special Imaging Diagnostics, Faculty of Dental Medicine, Medical University - Varna,

Tsar Osvoboditel 150, 9002 Varna.

Tel.: +359877922106

E-mail address: k.dzhabalyan@mu-varna.bg 